\title{
Towards precision radial velocity science with SALT's High-Resolution Spectrograph
}

Crause, Lisa, Kniazev, Alexei, Butler, R. Paul, Kuhn, Rudi, Roy, Arpita, et al.

Lisa A. Crause, Alexei Kniazev, R. Paul Butler, Rudi Kuhn, Arpita Roy, Keith Browne, Alrin Christians, Etienne Simon, Anthony Koeslag, Jonathan Love, Roufurd Julie, "Towards precision radial velocity science with SALT's HighResolution Spectrograph," Proc. SPIE 11447, Ground-based and Airborne Instrumentation for Astronomy VIII, 1144746 (13 December 2020); doi: $10.1117 / 12.2562004$

SPIE. Event: SPIE Astronomical Telescopes + Instrumentation, 2020, Online Only 


\title{
Towards precision radial velocity science with SALT's High-Resolution Spectrograph
}

\author{
Lisa A. Crause*a,b, Alexei Kniazevev, ${ }^{\mathrm{a}, \mathrm{b}}$, R. Paul Butler', Rudi Kuhn", ${ }^{\mathrm{a}, \mathrm{b}}$, Arpita Roy ${ }^{\mathrm{d}, \mathrm{e}}$, Keith Browne ${ }^{\mathrm{a}, \mathrm{b}}$, \\ Alrin Christians ${ }^{\mathrm{a}, \mathrm{b}}$, Etienne Simon ${ }^{\mathrm{a}, \mathrm{b}}$, Anthony Koeslag ${ }^{\mathrm{a}, \mathrm{b}}$, Jonathan Love ${ }^{\mathrm{a}, \mathrm{b}}$, Roufurd Julie ${ }^{\mathrm{a}, \mathrm{b}}$ \\ aSouth African Astronomical Observatory, P.O. Box 9, Observatory 7935, Cape Town, South Africa \\ ${ }^{b}$ Southern African Large Telescope, P.O. Box 9, Observatory 7935, Cape Town, South Africa \\ ${ }^{\mathrm{c}}$ Earth and Planets Laboratory, Carnegie Institution for Science, 5241 Broad Branch Road NW, \\ Washington D.C. 20015-1305, USA \\ dDepartment of Astronomy, California Institute of Technology, Pasadena, CA 91125, USA

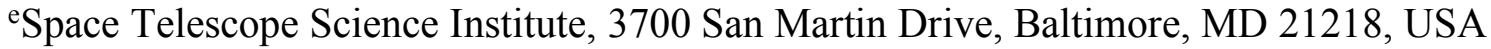

\begin{abstract}
We describe efforts to equip the Southern African Large Telescope (SALT) for precision radial velocity (PRV) work. Our current focus is on commissioning the high-stability (HS) mode of the High-Resolution Spectrograph (HRS), the mode intended to support exoplanet science. After replacing the original commercial iodine cell with a custom-built, precisely characterised one and following established best practice in terms of observing strategy and data reduction, this system now delivers $3-4 \mathrm{~m} / \mathrm{s}$ radial velocity stability on 5th and 6th magnitude stars. Unfortunately, the throughput is compromised by the HRS dichroic split being at $555 \mathrm{~nm}$ (i.e. roughly midway through the $100 \mathrm{~nm}$ span of the iodine absorption spectrum). Furthermore, SALT's fixed elevation axis limits the exposure time available for a given target and hence the depth and/or precision achievable with the iodine cell. The HS mode's simultaneous ThAr option uses the full 370-890 $\mathrm{nm}$ passband of the HRS and does not suffer gas cell absorption losses, so it may be more suitable for exoplanet work. The first step was to quantify the internal stability of the spectrograph, which requires simultaneously injecting arc light into the object and calibration fibres. The HS mode's optical feed was modified accordingly, stability test runs were conducted and the necessary analysis tools were developed. The initial stability test yielded encouraging results and though more testing is still to be done, SALT will likely pursue the development of a laser frequency comb to support the development of HRS PRV capability.
\end{abstract}

Keywords: Southern African Large Telescope (SALT), High Resolution Spectrograph (HRS), precision radial velocity (PRV), iodine cell, simultaneous ThAr, calibration, exoplanets, laser frequency comb

\section{INTRODUCTION}

The SALT ${ }^{1-8}$ HRS $^{9-10}$ has been in full science operation since 2014 and the three general-purpose (low-, medium- and highresolution) observing modes are scientifically productive, particularly following the introduction of data reduction pipelines in $2016^{11}$. The instrument also has a specialist high-stability (HS) mode, equipped with a fibre-double-scrambler, that makes provision for the sorts of precision wavelength calibration required for exoplanet research. Since SALT is keen to participate in PRV science, we have been working to commission the two options offered by the HS mode, namely an iodine cell, or the ability to inject thorium-argon light into a calibration fibre while observing a target with the object fibre (known as simultaneous ThAr). We reported on the iodine cell developments ${ }^{12}$ at the 2018 conference and here we provide an update on those efforts, before expanding on the work done subsequently to explore the simultaneous ThAr option.

*lisa@saao.ac.za

Ground-based and Airborne Instrumentation for Astronomy VIII, edited by Christopher J. Evans,

Julia J. Bryant, Kentaro Motohara, Proc. of SPIE Vol. 11447, 1144746 · (c) 2020

SPIE · CCC code: $0277-786 \mathrm{X} / 20 / \$ 21 \cdot$ doi: $10.1117 / 12.2562004$ 


\section{SALT HRS SUITABILITY FOR PRV SCIENCE}

A fundamental question associated with SALT's PRV aspirations is whether or not the HRS is suitable for this highly demanding application. If not, we would either have to consider developing a new dedicated instrument for exoplanet science, or give up on this exciting capability. We have therefore directed effort into commissioning and characterising the performance of the two precision wavelength calibration options offered by the HRS HS mode. The logic associated with this process is outlined in Fig. 1. We began with the iodine cell and took that as far as practically possible in a relatively short period of time, before moving on to exploring the potential of the simultaneous ThAr channel.

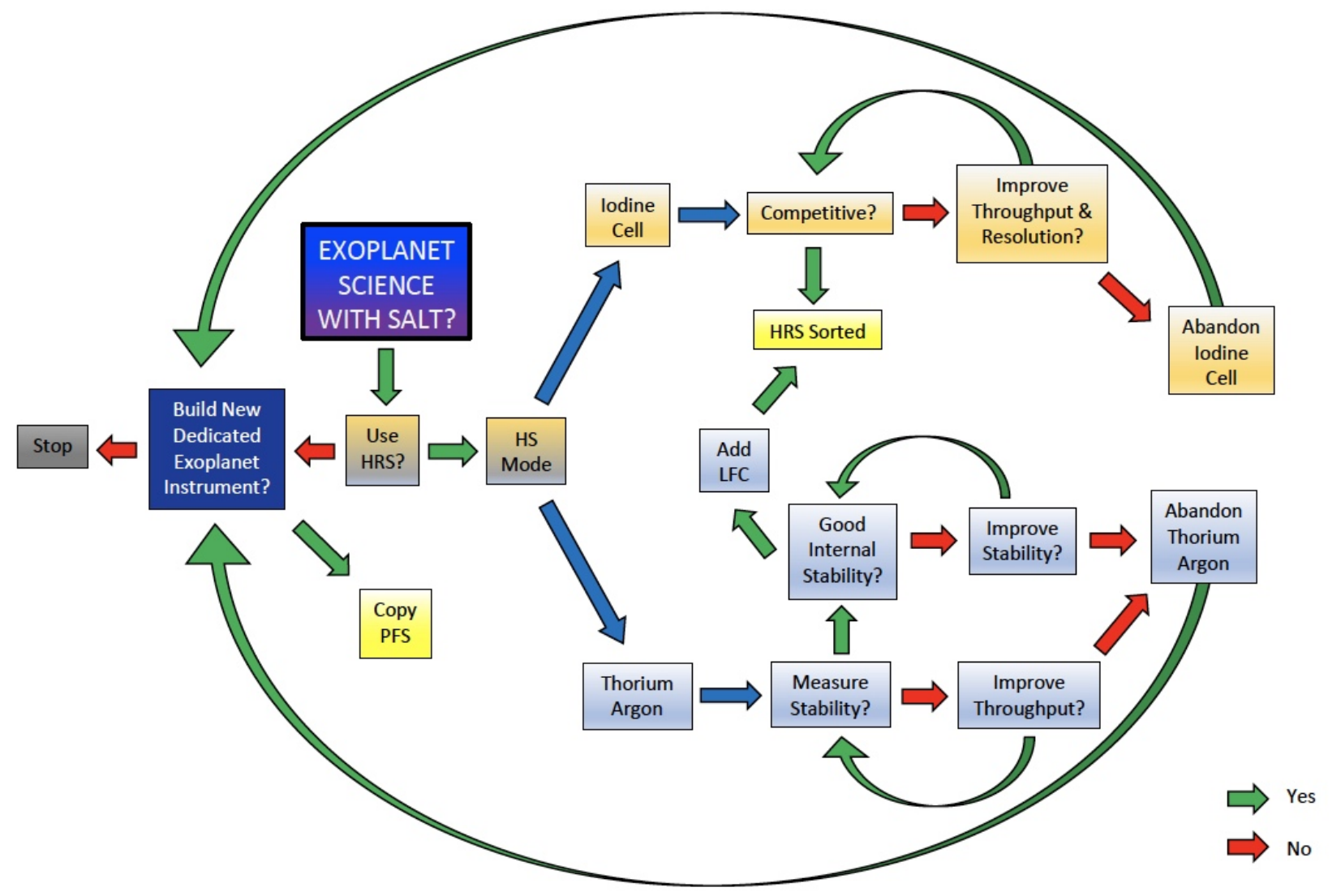

Figure 1. The logic employed in exploring whether the HRS's HS mode will be suitable for pursuing exoplanet science.

\section{IODINE CELL}

The original commercial iodine cell (which turned out to be intended for laser lab use) needed to be replaced with a custommade cell designed specifically for PRV science. The new cell had also been meticulously characterised at extremely high spectral resolution $\left(\mathrm{R} \sim 10^{6}\right)$ to provide the necessary detail in the iodine absorption spectrum. Integrating the new cell involved a significant overhaul of the cell housing and heating system (see Fig. 2), as well as changes to the HRS control software to render the system operationally safe and robust.

\subsection{Fibre-double-scrambler Re-alignment}

We did what was readily achievable to improve the iodine cell performance before collecting and analysing on-sky data. In particular, the fibre-double-scrambler (FDS) optics needed re-alignment, which called for opening the vacuum tank to allow light to be injected from the instrument side, not just from the focal plane. This is by far the most efficient way to re-align the fibres, as one can inject different colours of light in each end and then make the necessary adjustments. Light emerging from the lenses at the tank end form the targets (visible on a small piece of paper placed in the beam) onto which 
to position the spots from the adjustable input side of the FDS. Disruptive as it is to open the vacuum tank, we also had to address an intermittent problem with the mode selector mechanism, so we took advantage of that opportunity to do the realignment of the FDS at the same time.
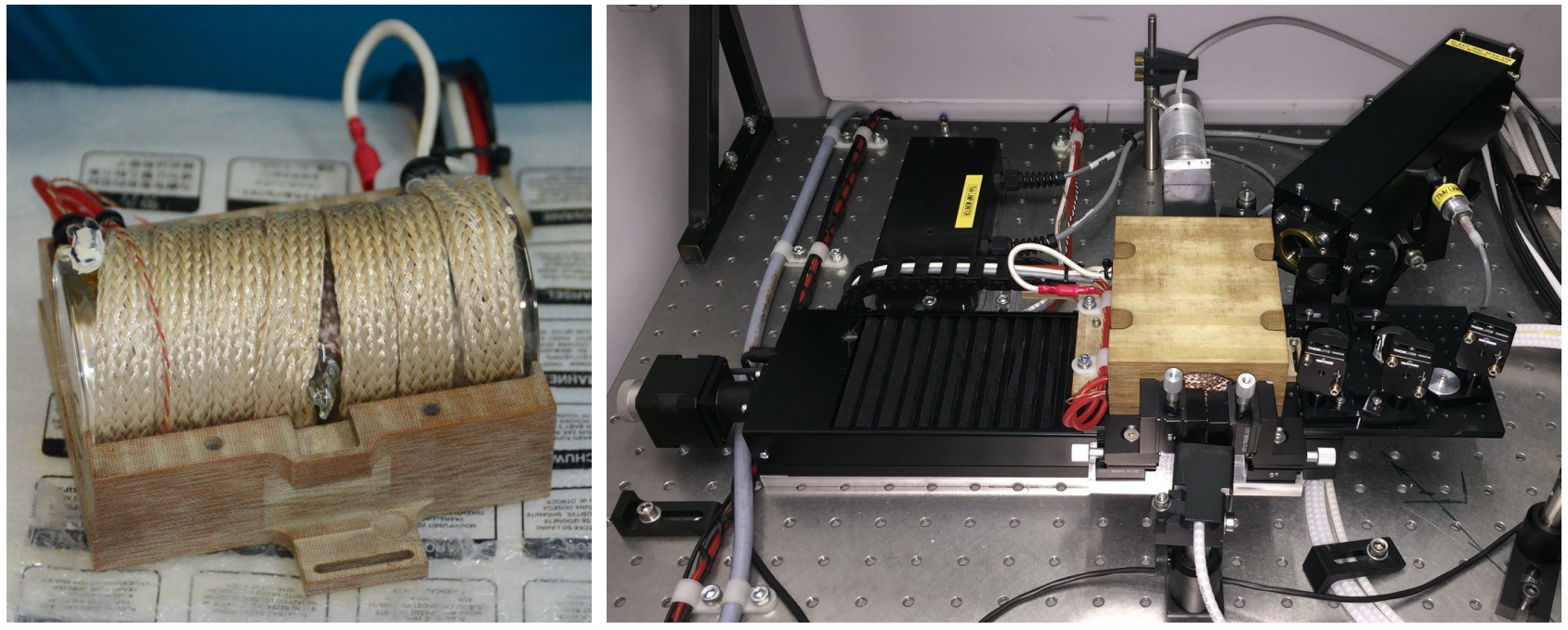

Figure 2. The new iodine cell surrounded by a heat wrap, connected to an extra thermocouple and a thermal fuse to serve as the final defence against overheating, all being installed in the cell's new 2-part housing (left). The iodine cell is mounted on the translation stage on the HS bench, which allows the cell to be positioned in the beam for iodine cell observations (right).

\subsection{On-sky Performance}

After the system had been tested and shown to be reliable and safe, we undertook various observations to establish the onsky performance of the new cell. The key result is the 3-4 m/s velocity stability measured for a known stable star, in this case the V=4.9 mag HD102365, over about a year (see Fig. 3).

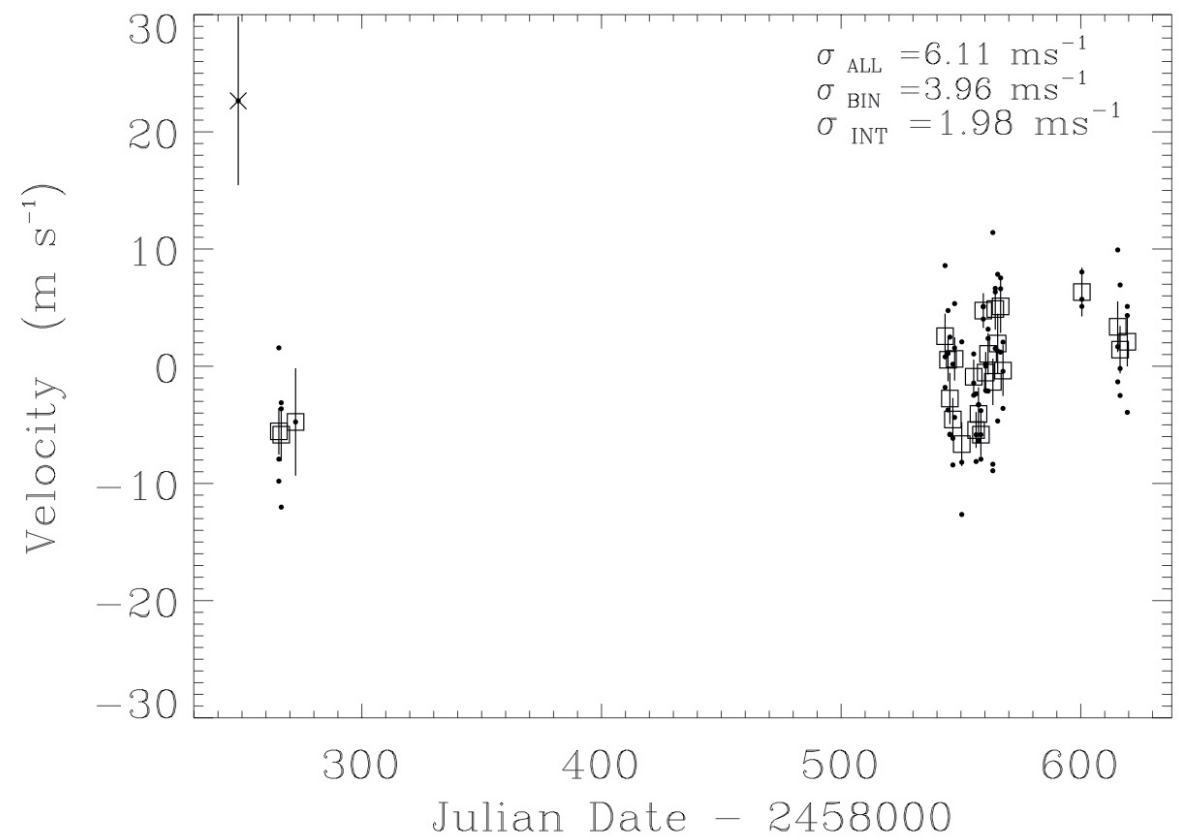

Figure 3. Radial velocity data for a bright (V=4.9 mag) stable star, HD102365, obtained with the iodine cell over the course of a year, indicating stability at the $3-4 \mathrm{~m} / \mathrm{s}$ level. 


\subsection{Potential Improvements}

We wanted to investigate the simultaneous ThAr option before committing additional resources to efforts to improve the iodine cell. But there are certain steps that could still be taken to extend the reach of the iodine cell for PRV science, including establishing optimal focus positions for the iodine regions in both the Red and the Blue channels. Making regular focus stage moves would add to observing overheads though, and could lead to reliability issues as the two focus stages currently remain fixed for all observations made with the other three HRS modes.

There are also more invasive interventions to consider that would benefit the entire instrument, such as replacing the fibre cable. This would allow us to shorten the 50-metre cable to a more appropriate length (the current cable routing is inefficient and could conceivably be reduced by $\sim 10 \%$ ) and to reduce stress-induced focal ratio degradation (FRD). We also have reason to believe that the fibre in the current cable may have poor intrinsic FRD and so using better fibre would be advantageous.

Even more ambitious would be the introduction of a fibre slicer for the HS mode, to eliminate the losses arising from the Bowen-Walraven slicer optics inside the vacuum tank. This would also allow for an increase in resolution for the HS mode, which would be beneficial for PRV science. Such a major modification would take this popular instrument offline for a significant amount of time, so the benefits would have to be carefully weighed against non-exoplanet science needs.

\section{SIMULTANEOUS THORIUM-ARGON}

The alternative to the iodine cell approach for PRV science is to have a highly stabilised spectrograph and inject arc light into a calibration fibre while observing a target in the object fibre. The original HRS system therefore made provision for injecting arc light from a ThAr lamp on the HS bench into either of the two HS mode fibres (individually) in support of such simultaneous ThAr observations (see Fig. 4). But the key consideration for this technique is the intrinsic stability of the instrument, which is best established by comparing velocity drifts measured between the object and calibration fibres. This requires having arc light down both fibres at once.

The only way to send arc light down both fibres simultaneously was to use the telescope's facility calibration system (calsys). This employs liquid light guides to convey light from lamps in the prime focus payload to the calibration screen that gets positioned just in front of the spherical aberration corrector. This light can then be directed to the fibre instrument port and into the HS mode fibres. Unfortunately, the combination of the relatively low calsys throughput and the length of the fibre cable makes this extremely inefficient. Calsys ThAr arcs for the HS mode take 600 seconds, which is not practical for the type of monitoring we needed to conduct.

\subsection{HS Bench Optical Feed Modification}
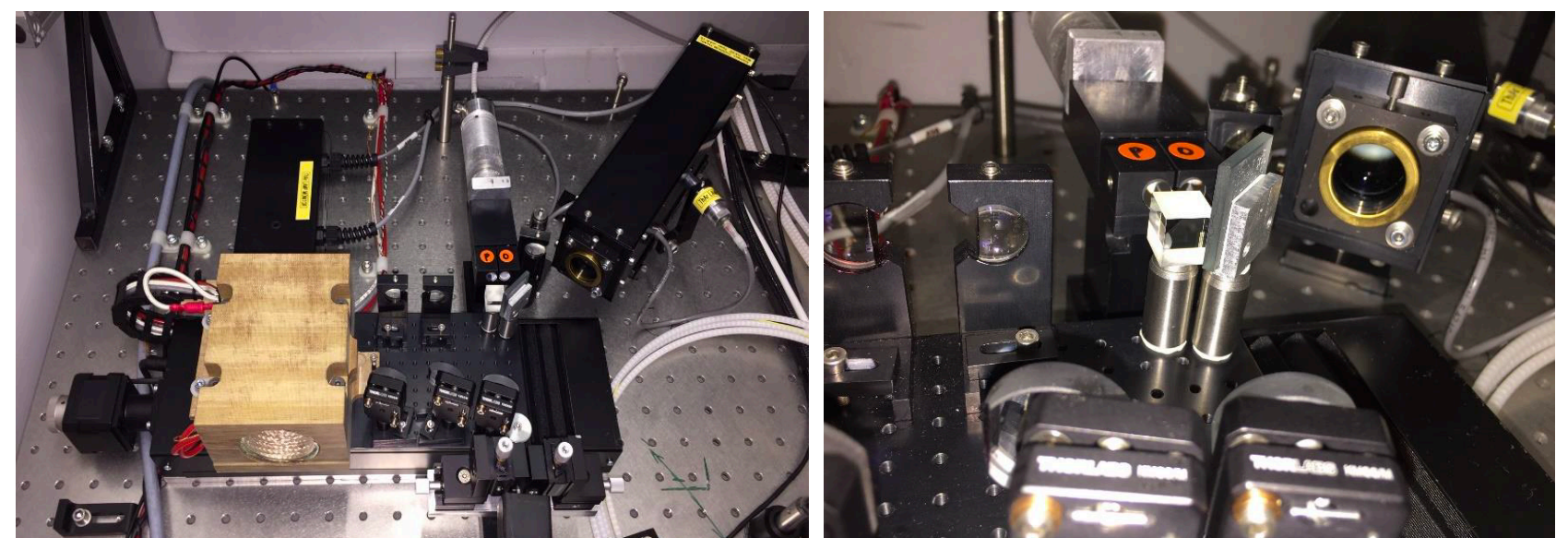

Figure 4. The HS bench configured to direct ThAr light (from the lamp in the upper right corner of the photos) into both fibres (labelled $\mathrm{P}$ and $\mathrm{O}$ ) at the same time (left). Close-up view of the new feed optics: an additional 50/50 beam-splitter and a small fold mirror placed just in front of the second set of fibre-double-scrambler lenses. 
Fortunately, the HS bench did have an extra station that was not used operationally. Its fold mirror could therefore be used to set up a new ThAr feed that only required the addition of a 50/50 beam-splitter and a small fold mirror to direct the reflected portion of the split beam into the second fibre. Eliminating the long run of fibre from prime focus and injecting the ThAr light almost directly from the lamp on the HS bench produces the required "reference arcs" with vastly more signal in a 90 second exposure than the calsys can deliver in a 10 minute exposure (see Fig. 5).
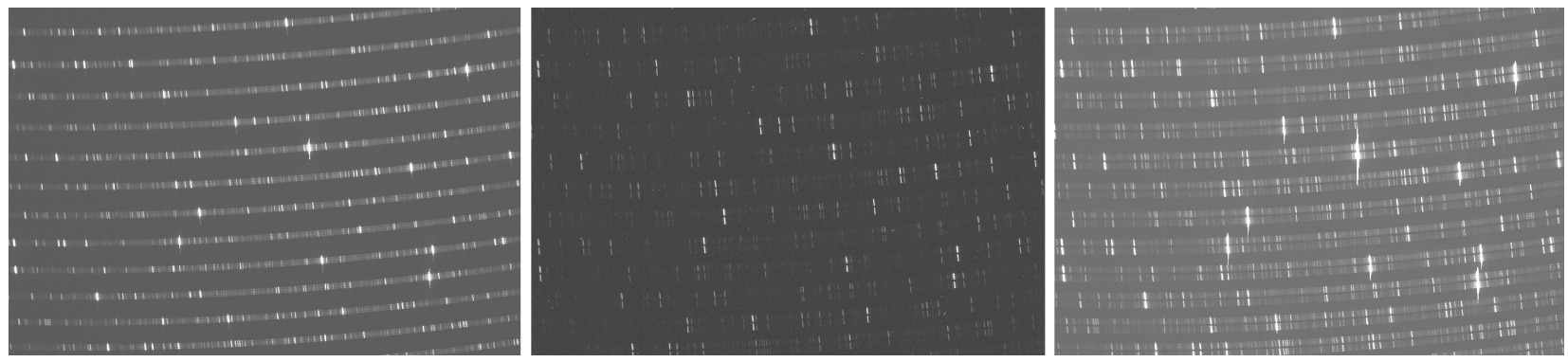

Figure 5. $90 \mathrm{sec}$ HRS Blue frame from the original single-fibre ThAr feed on the HS bench (left). A 600 sec HRS Blue frame of the SALT calibration system ThAr lamp (located near prime focus) directing arc light into both fibres at once (centre). 90 sec Blue "reference arc" frame obtained with the new optical feed that efficiently sends ThAr light from the lamp on the HS bench down both fibres simultaneously (right). These reference arcs are what were used for our stability analysis.

\subsection{Stability Analysis}

Using the new feed described above, we could inject equal amounts of light into both HS fibres at once and take 90 second exposures every 10 minutes over the course of two days (20 and 21 September 2019). This was done over seven and six hour intervals (respectively, over the two days). The telescope stood idle during the days but was fully operational during the night in between. This exercise resulted in $71 \mathrm{ThAr}$ exposures in total, for each of the Red and Blue channels of the HRS. Only the Blue data have been analysed so far; processing of the more challenging Red frames (that suffer severe saturation from a number of extremely bright argon lines) is currently in progress.

The basic approach was to explore the stability over time of all the unblended lines of suitable intensity ( $\sim 250$ in total, see Fig. 6) from the one fibre relative to all of those from the other fibre. The first frame was set as a reference and then the differences in line positions (for all of the usable lines in the first fibre) were calculated between each frame and the reference. The median/mean of those differences then represents the absolute drift of that fibre. The same procedure was followed for the second fibre, to establish its absolute drift. That absolute drift of the mean position of each fibre is shown in black (for the first fibre) and in blue (for the second) in the left panel of Fig. 7. Each fibre has an absolute drift with a mean value of $\sim 2.5 \mathrm{~m} / \mathrm{s}$ at the wavelength of the $\mathrm{H} \beta$ line (4861 $\AA$ ). The median shows the same tendency and amplitude.
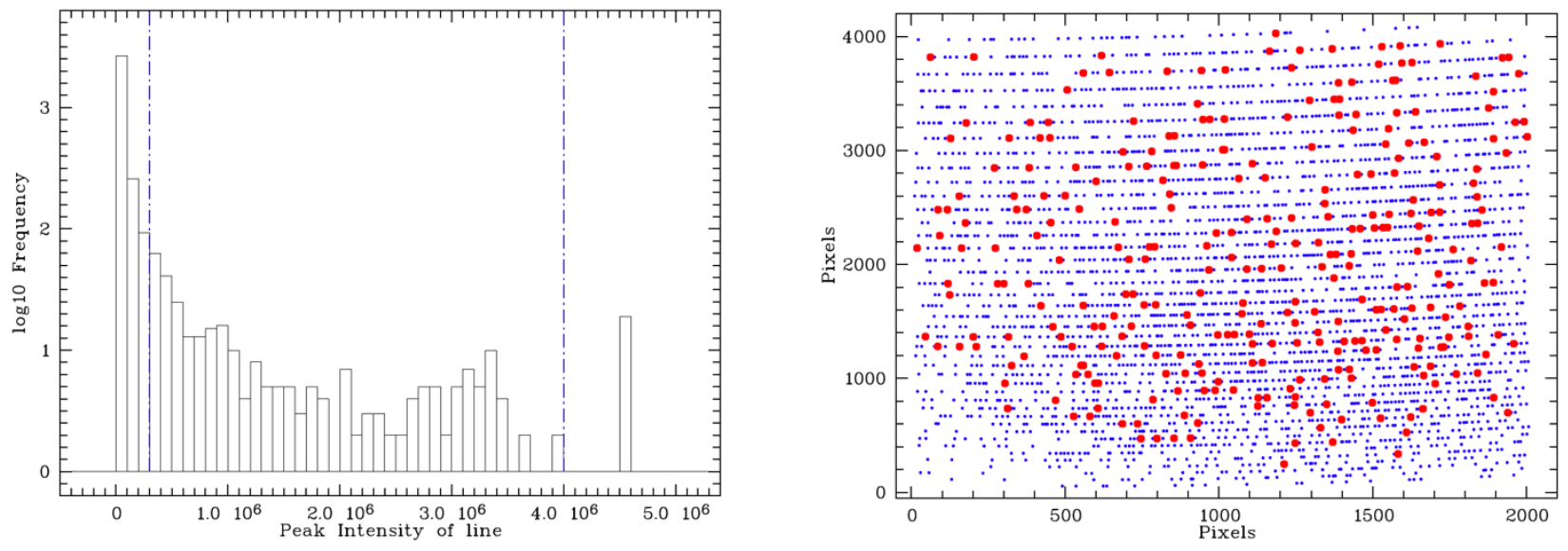

Figure 6. The range of peak intensities for all of the $\sim 3000$ lines identified in the Blue channel frames, of which $\sim 250$ within the blue dashed lines (intensities within the $1 \times 10^{5}-4 \times 10^{6}$ range) and not blended were selected for this analysis (left). All of the lines are shown in blue in the plot on the right, with those selected for the analysis marked in red. Note that the vertical separation of the two fibres is too small to be visible in the plot on the right, so each dot represents the lines in both fibres. 
Plotting the drift as a function of temperature (see the right panel in Fig. 7), using red and black points to distinguish the two days over which the test was run, reveals a clear trend that is well approximated by a second order polynomial.
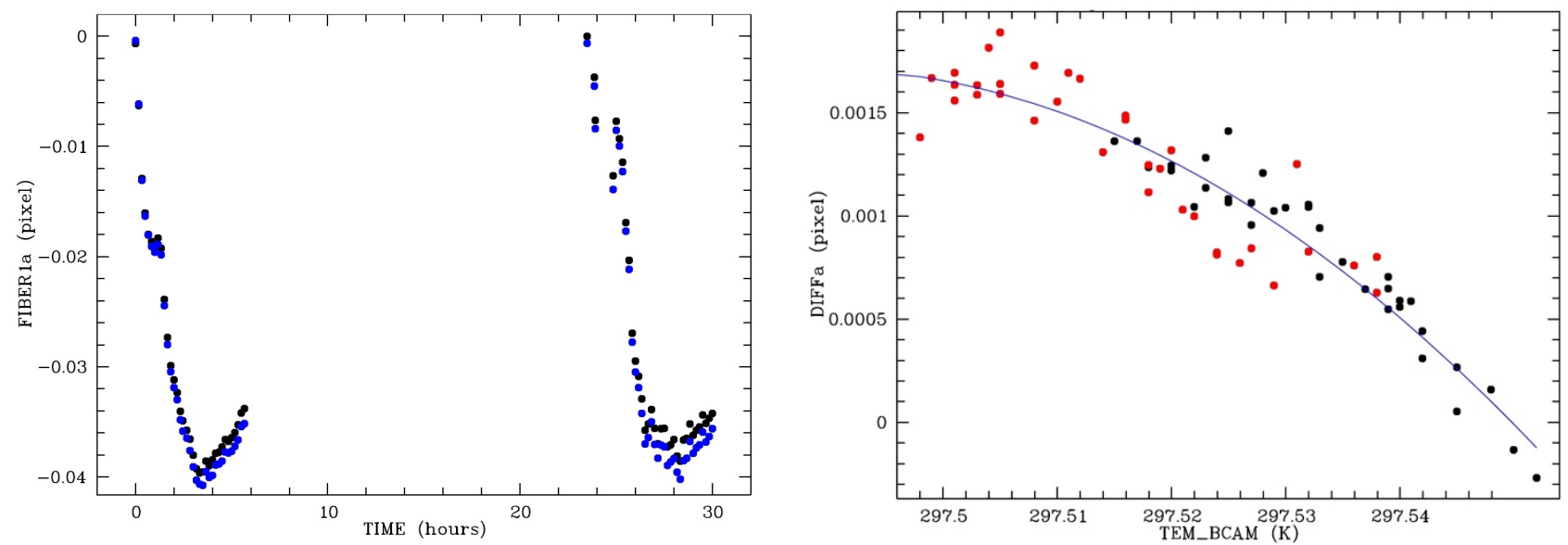

Figure 7. The plot on the left shows the absolute drift of the mean position of each fibre over the course of two days and the night in between (when the telescope was operational). Black symbols mark the mean position of the first fibre while blue symbols show the second fibre. The plot on the right shows the dependence of the instrument drift on the temperature measured on the lens barrel of the blue camera (located inside the vacuum tank). The red and black symbols are measurements from the two days over which the test was done and the blue curve is a second order polynomial fit to the points.

We then plot the difference between the drifts of the first and second fibres over time to show the overall instrument drift (see the black points in Fig. 8). Subtracting the temperature dependence then yields the blue points in Fig. 8, the distribution of which has a mean value of zero and an rms of 0.000152 pixel, or about $40 \mathrm{~cm} / \mathrm{s}$ at the wavelength of the $\mathrm{H} \beta$ line. These are highly encouraging results that justify further exploration of the simultaneous ThAr possibilities.

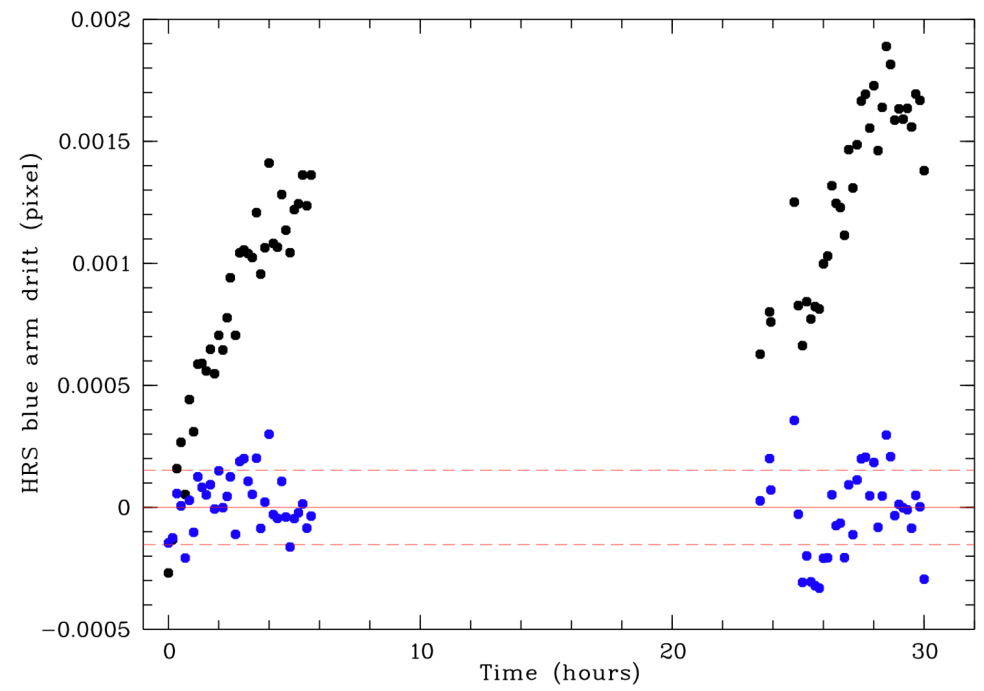

Figure 8. The black points show the instrumental drift over time, while the blue points show what remains after the temperature trend has been subtracted. The blue points have a mean velocity of zero with a $40 \mathrm{~cm} / \mathrm{s} \mathrm{rms} \mathrm{scatter.}$

\section{NEXT STEPS}

While the performance of the iodine cell is inherently limited by the spectrograph's dichroic split being roughly midway through the iodine wavelength range (and it would be effectively impossible to change this fundamental property of the instrument), we are more optimistic about the prospects presented by the HS mode's simultaneous ThAr option. 


\subsection{Complete the Stability Analysis}

The equivalent analysis for the Red channel data obtained during the initial stability test is currently in progress. While this is likely to produce very similar results to that obtained for the Blue channel, the comparison may offer some insights into the relative stability of the two cryostats and their influence on the overall instrumental drift. We are also eager to analyse the long-term monitoring data, consisting of daily reference arcs taken over the course of more than a year now. Further testing is needed, but we can already see an obvious way to improve the system's performance.

\subsection{Refine the HRS Temperature Control}

It is clear that we can significantly improve the temperature stability of the instrument by tuning the control loop for the heating tape attached to the outer surface of the tank. This is meant to servo the system to maintain a constant setpoint to within tens of milli-Kelvin. However, querying the various sensors in the SALT event logging system shows that the tank temperature is currently not being well controlled, which in turn has a predictable influence on the components inside the tank. Fig. 9 shows temperature data for a typical week, with the outer surface of the tank (blue curve) varying by more than a tenth of a degree and the collimator mount (inside the tank, shown in red) responding to those variations.

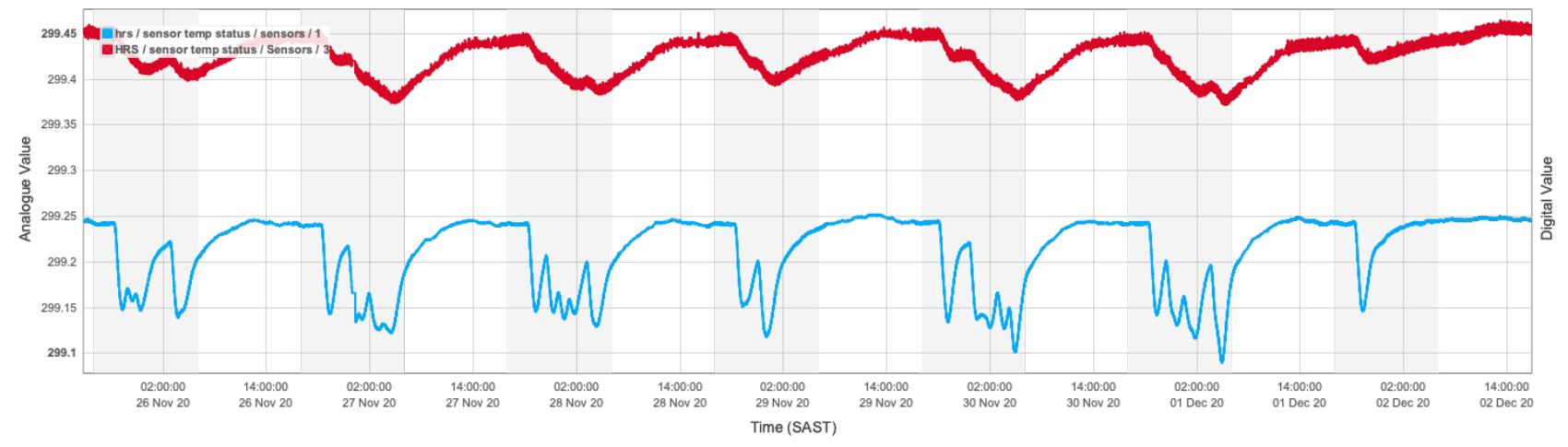

Figure 9. A week's worth of temperature data for the outside of the HRS vacuum tank (blue) and the collimator mount on the optical bench inside the tank (red). Having the tank temperature varying by more than a tenth of a degree drives smaller, but still highly significant, temperature variations inside the tank.

\subsection{Develop Software Tools for Simultaneous ThAr Observations}

The HRS pipeline only serves the LR, MR and HR modes, but not the more demanding HS one. We therefore need to set up the appropriate software tools to deal with simultaneous ThAr observations. The throughput of the HS mode is about $5 \%$, approximately half that of $\mathrm{HR}$, but we have yet to determine the limiting magnitude using on-sky data. Once we have the necessary data reduction tools, we will attempt to recover a selection of known exoplanets to demonstrate this capability with the HRS.

\subsection{Investigate and Pursue Laser Frequency Comb Options}

Our previous experience in temporarily hosting an experimental laser frequency comb (LFC) ${ }^{13}$ at SALT showed the enormous potential associated with this remarkable precision wavelength calibration device. It was immediately obvious how much better a LFC is compared to a ThAr lamp - producing orders of magnitude more lines, equally spaced in frequency, neither blended nor differing widely in intensity and fully traceable over time (see Fig. 10).

However, at that stage (in mid-2016) the SALT community showed minimal interest in exoplanet science and we knew relatively little about the HS mode of the HRS. Interest in pursuing PRV science (particularly as a possible way to attract new partners into the SALT consortium) has subsequently increased and so the question that needed to be answered was whether this general-purpose facility instrument is stable enough to justify investing in a LFC in support of SALT's PRV ambitions.

The stability that we have seen so far, in spite of the large temperature variations that the vacuum tank has been subject to, suggests that the HRS is intrinsically stable enough to benefit from the addition of a LFC. Therefore, we are investigating 
possibilities for developing a LFC that would be appropriate for SALT. This would not need to be a turn-key system, as we welcome the opportunity to work with LFC specialists and get to learn about these devices through engaging with the hardware, as we did during the 2016 LFC "field trial".
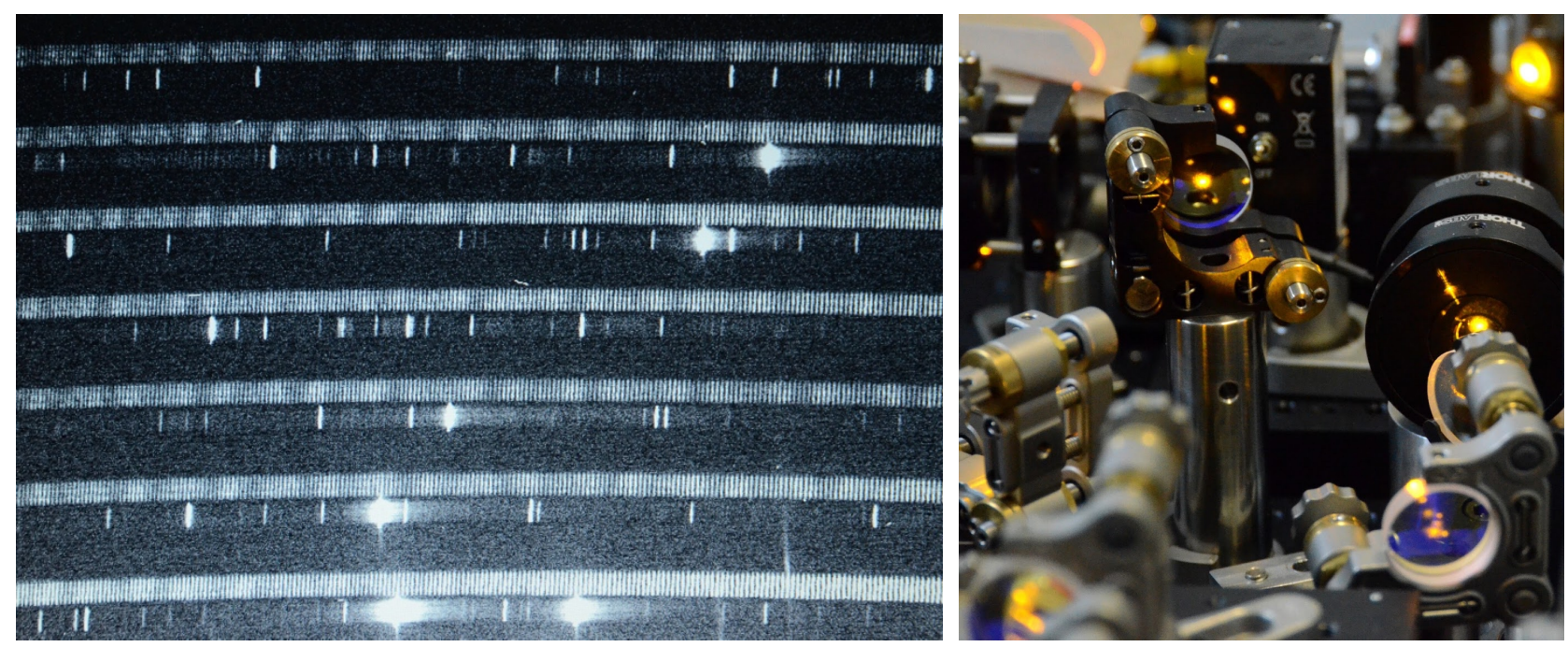

Figure 10. The striking comparison between the experimental LFC (upper track) and the HS bench ThAr lamp (lower track) in the Red channel of the HRS (left). Some of the components of the Heriot-Watt University LFC that we tested at SALT in 2016 (right).

We would start with a comb that only spans the Red channel of the HRS (555-890 nm), which is far simpler than having to delve into the blue spectral region that is extremely challenging for LFCs. Conveniently, the Blue channel of the HRS happens to be relatively well served by the ThAr lamp, while the Red beam is plagued by a number of problematic argon lines that saturate dramatically. We deal with this by filtering the lamp light, which in turn eliminates a number of otherwise useful lines in the redder half of the Red channel, as shown in Fig 11. Starting with a Red channel comb will therefore be most beneficial and then we would plan to incorporate additional wavelength ranges (starting with frequency doubling to access the $400 \mathrm{~nm}$ region) at a later stage, as funding permits and LFC technology continues to evolve.
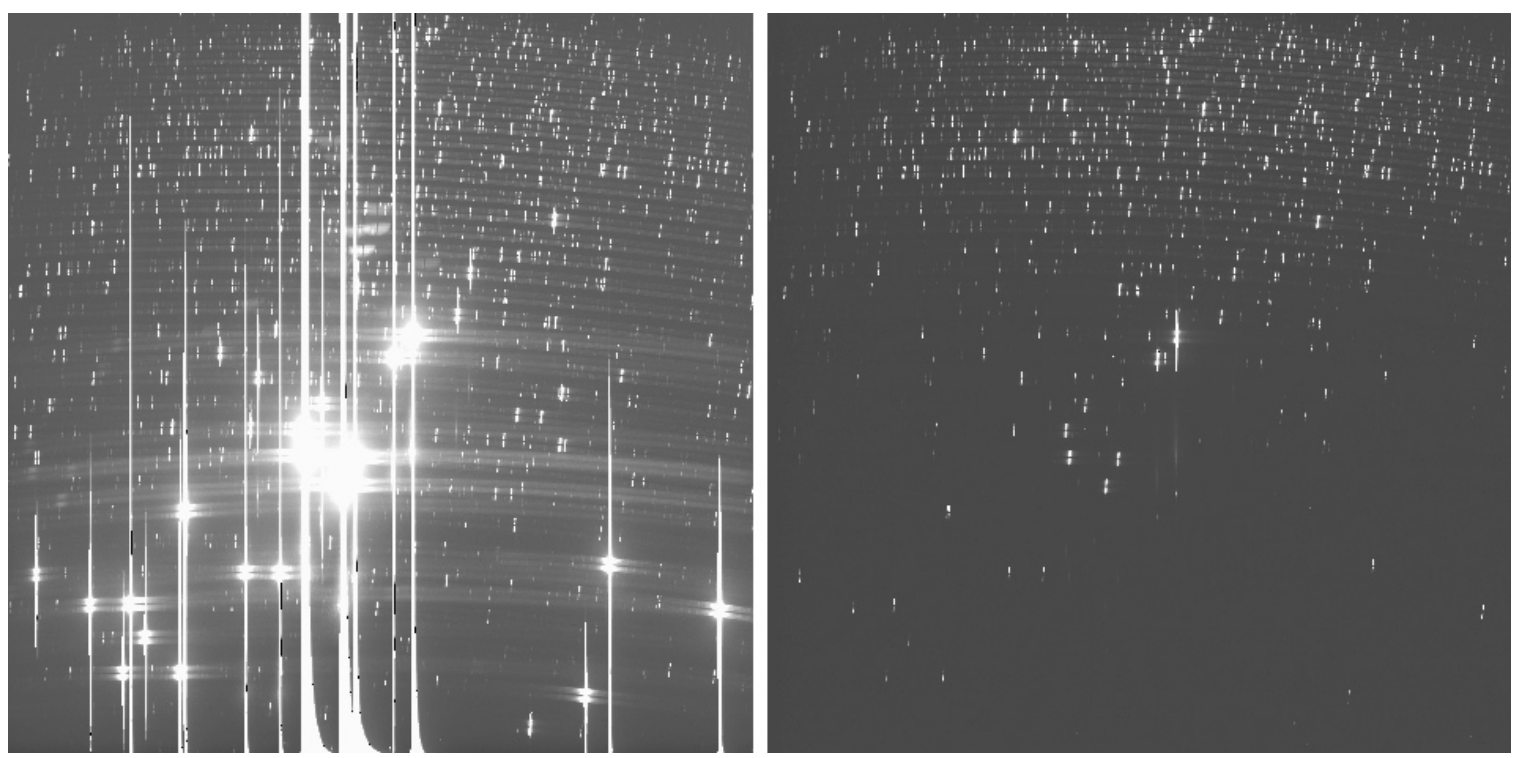

Figure 11. Comparison between HRS Red channel ThAr "reference arc" frames, without filtering of the lamp (left) and with filtering (right) to suppress the strong argon lines that saturate and bleed across the frames. 


\section{CONCLUSION}

We have a far better understanding of the HS mode now and refining the vacuum tank temperature controller performance will dramatically improve the instrument stability. Any interventions to increase the throughput (such as replacing the fibre cable) will benefit all of the modes, particularly the iodine cell. Lastly, the addition of a LFC for the Red channel of the HRS will enhance the velocity precision achievable with the HS mode. While there is still much to be done to make competitive PRV science with SALT HRS a reality, we are excited to have made significant progress in this direction.

\section{ACKNOWLEDGMENTS}

We acknowledge the National Research Foundation of South Africa and the Southern African Large Telescope Foundation for support for this work. The observations reported in this paper were obtained with the Southern African Large Telescope (SALT). We gratefully acknowledge the detailed discussions with Richard McCracken (of the UltraFast Optics group at Heriot-Watt University in Edinburgh) regarding laser frequency combs and options that may be suitable for SALT HRS. Most significantly, we wish to acknowledge the superb effort by the team at Durham University's Centre for Advanced Instrumentation that produced the HRS. They designed and built a general-purpose high-resolution spectrograph, most of a decade ago, that successfully incorporated many of the tricks of the PRV trade that are still in use in instruments being built today. Not that we doubted you, but it just seemed too good to be true given the demands of this particular application!

\section{REFERENCES}

[1] Stobie, R., Meiring, J. G., Buckley, D. A. H., "Design of the Southern African Large Telescope (SALT)", Proc. SPIE 4003, 355 (2000).

[2] Swat, A., O'Donoghue, D., Swiegers, J., Nel, L. and Buckley, D. A. H., "The optical design of the Southern African Large Telescope", Proc. SPIE 4837, 564 (2003).

[3] Meiring, J. G., Buckley, D. A. H., "Southern African Large Telescope (SALT) project, progress and status after 4 years", Proc. SPIE 5489, 592 (2004).

[4] O'Donoghue, D. et al., "First science with the Southern African Large Telescope: peering at the accreting polar caps of the eclipsing polar SDSS J015543.40+002807.2", Mon. Not. R. Astr. Soc, 372, 151 (2006).

[5] O'Donoghue, D., "Correction of spherical aberration in the Southern African Large Telescope (SALT)", Proc. SPIE 4003, 363 (2000).

[6] O’Donoghue, D. E., O’Connor, J. E., Crause, L. A., Strümpfer, F., Strydom, O. J., Brink, J. D., Sass, C., Wiid, E., Atad-Ettedgui, E., "Saving SALT: repairs to the spherical aberration corrector of the Southern African Large Telescope (SALT)", Proc. SPIE 7739, 21 (2010).

[7] Crause, L. A., O’Donoghue, D. E., O’Connor, J. E., Strümpfer, F., Strydom, O. J., Sass, C., du Plessis, C., Wiid, E., Love, J., Brink, J. D., Wilkinson, M., Coetzee, C., “A happy conclusion to the SALT image quality saga”, Proc. SPIE 8444, 8444I (2012).

[8] http://www.salt.ac.za

[9] Bramall, D. G., Sharples, R., Tyas, L., Schmoll, J., Clark, P., Luke, P., Looker, N., Dipper, N. A., Ryan, S., Buckley, D. A. H., Brink, J., Barnes, S. I., "The SALT HRS spectrograph: final design, instrument capabilities, and operational modes", Proc. SPIE 7735, 77354F (2010).

[10] Crause, L. A., Sharples, R. M., Bramall, D. G., Schmoll, J., Clark, P., Younger, E. J., Tyas, L. M. G., Ryan, S. G., Brink, J. D., Strydom, O. J., Buckley, D. A. H., Wilkinson, M., Crawford, S. M., and Depagne, É., "Performance of the Southern African Large Telescope (SALT) High Resolution Spectrograph (HRS)", Proc. SPIE 9147, 91476T (2014).

[11] Crawford, S. M., Crause, L. A., Depagne, É., Iłkiewicz, K., Schroeder, A., Kuhn, R., Hettlage, C., RomeroColmenero, E., Kniazev, A., Väisänen, P., "Data Reductions and Data Quality for the High Resolution Spectrograph on the Southern African Large Telescope", Proc. SPIE 9908, 99082L (2016).

[12] Crause, L. A., Butler, R. P., Nave, G., Kuhn, R., Lomberg, B., Kniazev, A., Crawford, S. M., Depagne, É., "Commissioning the SALT High Resolution Spectrograph's Iodine Cell", Proc. SPIE 10702, 107025S (2018).

[13] Depagne, É., McCracken, R. A., Reid, D. T., Kuhn, R. B., Erasmus, N., Crause, L. A., "First light of a laser frequency comb at SALT", Proc. SPIE 9908, 99087M (2016). 